\title{
Conciliación y revisión de la medicación al alta en paciente sometido a gastrostomía para alimentación enteral
}

\author{
Francesc Xavier Moranta Ribas \\ Licenciado en Farmacia. Farmacéutico comunitario en Palma (Mallorca).
}

\section{PALABRAS CLAVE}

Conciliación de la medicación, Revisión del uso de la medicación, gastrostomía endoscópica percutánea

\section{ABREVIATURAS}

EFG: especialidad farmacéutica genérica

PRM: problemas relacionados con los medicamentos

RNM: resultados negativos asociados a la medicación

\section{KEYWORDS}

Medication reconciliation, Medication use review, percutaneous endoscopic gastrostomy

\section{Presentación}

Hombre de 74 años que presenta múltiples patologías. Su esposa acude a la farmacia con informe de alta hospitalaria con el fin de recoger la medicación del paciente. El motivo del ingreso ha sido la realización de una gastrostomía endoscópica percutánea (GEP) como vía para instaurar la nutrición enteral debido a una patología esofágica. Según las instrucciones indicadas en el informe de alta, el paciente debe seguir con el mismo tratamiento, dosis y pauta posológicas, aunque evidentemente no la vía de administración previa a su hospitali- zación (tabla 1). La forma de administración de los medicamentos que le han indicado por escrito en el mismo informe es la de pulverizar en un mortero todos los medicamentos conjuntamente, con la excepción de las cápsulas que deben abrirse y pulverizar su contenido con el resto, mezclar el polvo resultante con agua e introducir la suspensión mediante jeringa por la sonda según la pauta posológica establecida. De esta forma lo están haciendo desde la implantación de la nueva vía. La esposa del paciente nos comenta que aunque la intervención e implantación del estoma se realizaron

Tabla 1 Tratamiento al alta hospitalaria

\begin{tabular}{|l|l|c|l|c|}
\hline Marca & \multicolumn{1}{|c|}{ P.A. } & Dosis & \multicolumn{1}{|c|}{$\begin{array}{c}\text { Forma } \\
\text { farmacéutica }\end{array}$} & Pauta \\
\hline Esomeprazol EFG & Esomeprazol & $40 \mathrm{mg}$ & Cápsulas & $1 / 24 \mathrm{~h}$ \\
\hline Adiro & A.A.S & $100 \mathrm{mg}$ & $\begin{array}{l}\text { Comprimidos } \\
\text { recubiertos }\end{array}$ & $1 / 24 \mathrm{~h}$ \\
\hline Uniket retard & $\begin{array}{l}\text { Mononitrato } \\
\text { de isosorbida }\end{array}$ & $50 \mathrm{mg}$ & $\begin{array}{l}\text { Comprimidos } \\
\text { liberación } \\
\text { prolongada }\end{array}$ & $1 / 24 \mathrm{~h}$ \\
\hline Duodart & $\begin{array}{l}\text { Dudasterida/ } \\
\text { tamsulosina }\end{array}$ & $0,5 / 0,4 \mathrm{mg}$ & $\begin{array}{l}\text { Capsulas } \\
\text { microencapsuladas }\end{array}$ & $1 / 24 \mathrm{~h}$ \\
\hline Emcomcor & Bisoprolol & $2,5 \mathrm{mg}$ & Comprimidos & $1 / 24 \mathrm{~h}$ \\
\hline Acovil & Ramipril & $5 \mathrm{mg}$ & Comprimidos & $1 / 24 \mathrm{~h}$ \\
\hline Simvastatina EFG & Simvastatina & $20 \mathrm{mg}$ & Comprimidos & $1 / 24 \mathrm{~h}$ \\
\hline
\end{tabular}

Este informe de caso clínico fue presentado en forma de comunicación oral al VIII Congreso de Farmacéuticos Comunitarios celebrado en Alicante en mayo de 2018, recibiendo el premio al mejor caso clínico presentado a dicho congreso.

Financiación: ninguna.

Conflicto de intereses: ninguno.

Cite este artículo como: Moranta FX. Conciliación y revisión de la medicación al alta en paciente sometido a gastrostomía para alimentación enteral. Farmacéuticos Comunitarios. 2018 Sep 28; 10(3):32-34. doi:10.5672/FC.2173-9218. (2018/Vol10).003.05

Correspondencia: Francesc Xavier Moranta Ribas (farmacia@farmaciasanz.com)

ISSN 1885-8619 @SEFAC (Sociedad Española de Farmacia Familiar y Comunitaria). Todos los derechos reservados. 
sin incidencias y se le dio el alta a las 48 horas, el paciente refiere dolor epigástrico y otros síntomas compatibles con gastritis desde unos días después de empezar a administrar el tratamiento por la nueva vía, nos comenta también que de forma repetida se les obstruye la sonda al administrar el tratamiento farmacológico.

\section{Evaluación}

El farmacéutico observa a simple vista que ciertos tratamientos del paciente son incompatibles con las instrucciones de administración recibidas y algunos incluso con la administración por vía enteral. Es decir, parece ser que no se ha realizado la conciliación al alta del tratamiento crónico para la nueva vía de administración. Se indica a la esposa del paciente que se hace necesaria la revisión del tratamiento completo con el fin comprobar su idoneidad para esta nueva forma de administración de fármacos. Para este fin es imprescindible que el farmacéutico realice una actualización de conocimientos sobre la implantación y uso de sondas gástricas percutáneas, ya que al ser prácticas quirúrgicas poco comunes y con baja prevalencia en la población existe un desconocimiento general sobre esta temática. Por otro lado, se hace patente la necesidad de búsqueda y consulta de bibliografía general sobre esta materia, así como de guías específicas de administración de medicamentos por sonda, más allá de la ficha técnica de cada medicamento implicado en el caso [1-4]. Una vez realizada la actualización de conocimientos sobre esta temática se revisa el tratamiento completo usando las fuentes bibliográficas antes mencionadas. Se detectan posibles Problemas Relacionados con los Medicamentos (PRM) que podrían conducir a Resultados Negativos asociados a la Medicación (RNM) de seguridad y eficacia [5].

\section{Intervención}

Tras la revisión se realizan las siguientes recomendaciones por escrito con el fin de hacerlas llegar al médico de familia o especialista específico, resaltando los problemas que causan resultados negativos de inseguridad e inefectividad detectados:
- Esomeprazol EFG: debería administrarse en forma de comprimidos, que se pueden pulverizar y disolver en agua, no en cápsulas, ya que en ese caso tendrían que abrirse y disolverse los microgránulos que contienen en una solución ácida. En ningún caso abrirse y pulverizar su contenido como se estaba haciendo, ya que de este modo disminuye su absorción y en consecuencia su eficacia.

- Adiro ${ }^{\circledR}$ (AAS): no se recomienda por esta vía por su cubierta entérica. $\mathrm{Al}$ eliminar esta cubierta como se estaba haciendo al pulverizarlo se incrementaba el riesgo de efectos adversos a nivel gastrointestinal. Una opción sería sustituirlo por Tromalyt ${ }^{\circledR}$ (AAS) 150 mg, ya que las cápsulas pueden abrirse y su contenido mezclarse con agua, pero en ningún caso deben triturarse los microgránulos que contienen.

- Uniket Retard ${ }^{\circledR}$ (Mononitrato de isosorbida): al ser de liberación prolongada es totalmente incompatible con esta vía. Al pulverizarse, como se estaba haciendo, se alteraba completamente su farmacocinética afectando a su eficacia. Existen dos posibles alternativas, el uso de mononitrato de isosorbida en comprimidos de liberación inmediata cada 8-12 h o el uso de parches de nitroglicerina del tipo y dosis que crea más adecuado el especialista.

- Duodart ${ }^{\circledR}$ (Dutasterida/Tamsulosina): es incompatible con esta vía al estar formulado en forma de cápsulas microencapsuladas de liberación prolongada, como también ocurre con las presentaciones comercializadas de tamsulosina. La única alternativa viable sería el uso de alfuzosina en comprimidos, uno cada $12 \mathrm{~h}$; aunque sean recubiertos en este caso en concreto se pueden pulverizar y dispersar en agua. $\mathrm{Al}$ abrirse las cápsulas y pulverizar su contenido se estaba comprometiendo totalmente la eficacia de este medicamento.

El resto del tratamiento, Emcom$\operatorname{cor}^{\circledR}$ (bisoprolol), Acovil ${ }^{\circledR}$ (ramipril) y simvastatina EFG son compatibles con esta vía y forma de administración.

Además de las recomendaciones específicas a los facultativos sobre el tratamiento, se instruye a la cuidadora del paciente en las normas de administración general de medicamentos por sonda, que básicamente son que deben administrarse cada uno de ellos por separado, con su propia jeringa disolviéndolo directamente 0 en forma de suspensión con 10-20 cc de agua estéril. Por norma general, a falta de revisar las posibles interacciones medicamento-alimento, deberían administrarse una hora antes o dos después de la administración de la alimentación enteral y solamente pulverizarse en mortero los que así hayamos indicado; en este caso en concreto bisoprolol, ramipril y simvastatina a la espera de los resultados de la intervención farmacéutica.

\section{Resultados}

La intervención del farmacéutico fue aceptada, el médico de familia y el cardiólogo instauraron todos los cambios aconsejados en el tratamiento (tabla 2). Con las nuevas instrucciones de administración de los fármacos en la revisión de la medicación, y tras la conciliación del tratamiento, la sonda ya no se obstruyó desapareciendo en pocos días las molestias gástricas.

Tabla 2 Tratamiento después de la intervención farmacéutica

\begin{tabular}{|l|l|l|l|c|}
\hline Marca & \multicolumn{1}{|c|}{ P.A. } & \multicolumn{1}{|c|}{ Dosis } & Forma farmacéutica & Pauta \\
\hline Esomeprazol EFG & Esomeprazol & $40 \mathrm{mg}$ & Comprimidos & $1 / 24 \mathrm{~h}$ \\
\hline Tromalyt & A.A.S & $150 \mathrm{mg}$ & $\begin{array}{l}\text { Cápsulas } \\
\text { microencapsuladas }\end{array}$ & $1 / 24 \mathrm{~h}$ \\
\hline Minitran & $\begin{array}{l}\text { Trinitrato de } \\
\text { glicerilo }\end{array}$ & $10 \mathrm{mg} / 24 \mathrm{~h}$ & Parches & $1 / 24 \mathrm{~h}$ \\
\hline Benestan & Alfuzosina & $2,5 \mathrm{mg}$ & Comprimidos & $1 / 12 \mathrm{~h}$ \\
\hline Emcomcor & Bisoprolol & $2,5 \mathrm{mg}$ & Comprimidos & $1 / 24 \mathrm{~h}$ \\
\hline Acovil & Ramipril & $5 \mathrm{mg}$ & Comprimidos & $1 / 24 \mathrm{~h}$ \\
\hline Simvastatina EFG & Simvastatina & $20 \mathrm{mg}$ & Comprimidos & $1 / 24 \mathrm{~h}$ \\
\hline
\end{tabular}

En rojo todos los cambios efectuados después de la conciliación en el tratamiento del paciente. 


\section{Conclusiones}

La conciliación al alta de la medicación es un servicio que compete plenamente al farmacéutico del nivel asistencial en el que se encuentre. Al ser una intervención relativa al ámbito hospitalario parecería lógico pensar que sería competencia exclusiva del farmacéutico de hospital, aunque en la práctica diaria como se nos demuestra en este caso, se hace imprescindible contar con la figura del farmacéutico comunitario. El farmacéutico comunitario debería ser incluido en los circuitos de conciliación de la medicación al alta hospitalaria y ser un actor de referencia en la continuidad asistencial.

\section{Referencias bibliográficas}

1. Fichas técnicas de medicamentos. Base de Datos de medicamentos del Consejo General de Farmacéuticos (Bot PLUS 2.0); 2016 [acceso 14 de mayo de 2016]. Disponible en: https://botplusweb.portalfarma.com

2. Peña Pedrosa JA, Santiago Pérez A. Guía de administración de medicamentos por sonda de alimentación enteral. [Monografía en internet]. Servicio de Farmacia. Hospital Clínico San Carlos; 2012 [acceso 14 de mayo de 2016]. Disponible en: http://www. madrid.org/cs/Satellite?blobcol=urldatactblobheader $=$

3. Gómez López L, Pedrón Giner C, Martínez Costa C. Guía para la administración y los cuidados de la Nutrición En- teral a través de Sonda Nasogástrica. Sociedad Española de Gastroenterología, Hepatología y Nutrición pediátrica; 2013 [acceso 14 de mayo de 2016]. Disponible en: https://www.seghnp. org/documentos/guia-para-la-administracion-y-los-cuidados-de-la-nutricion-enteral-traves-de-sonda

4. Administración de medicamentos en pacientes con nutrición enteral mediante sonda. Nutr Hosp [Internet] 2006. [acceso 14 de mayo de 2016];21(Supl. 4):139-97. Disponible en: http://www.nutricionhospitalaria. com/pdf/revista/309.pdf

5. Foro de Atención Farmacéutica, panel de expertos. Documento de Consenso, Enero de 2008. Ed. Consejo General de Colegios Oficiales de Farmacéuticos, Madrid. ISBN 978- 84-691-1243-4 